\title{
Softwares para automação de bibliotecas e centros de documentação na literatura brasileira até 1998
}

Gercina Ângela Borém Lima

\section{Resumo}

Estudo panorâmico sobre a utilização de software para a automação de bibliotecas no Brasil até 1998, contendo análise de tendências desta produção bibliográfica quanto à sua autoria, tipo de documentação, meio de publicação, distribuição geográfica e no tempo. Inclui uma descrição sucinta dos principais software citados na literatura.

\section{Palavras-chave}

Software para automação de bibliotecas; Gerenciadores de bases de dados bibliográficas; Tendências da publicação bibliográfica; Documentação.

\section{INTRODUÇÃO}

O objetivo deste trabalho é estudar a produção bibliográfica sobre os softwares que têm sido utilizados na automação de bibliotecas ou de sistemas bibliográficos no Brasil. Ao analisar comparativamente o conteúdo dessa literatura, avaliar sua relevância e descrever esses softwares sucintamente, objetivou-se criar uma fonte de referência e ponto de partida para estudos mais aprofundados. Pretendeu-se, também, diminuir a carência de materiais didáticos atualizados na área de tecnologia aplicada ao tratamento da informação, provendo os alunos de informações básicas sobre os programas de computador mais utilizados em bibliotecas no Brasil, bem como seu funcionamento.

Foi considerada aqui a literatura nacional publicada em revistas especializadas e anais de eventos científicos de biblioteconomia e documentação a partir de $1968^{*}$. Os artigos que tratavam da automação de bibliotecas de maneira geral não foram considerados, por entender-se que uma abordagem mais abrangente fugiria do objetivo principal deste trabalho. Inicialmente, a bibliografia levantada totalizou 98 referências, que foram divididas em três grupos: 75 são textos que se referem a softwares para automação de bibliotecas, centros de documentação, serviços de informação ou catalogação automática; 18 artigos tratam de conceitos gerais de indexação automática ou suas aplicações; os 5 textos restan-

* O recorte cronológico a partir de 1968 devese ao fato de que é desse ano a primeira experiência brasileira em serviços automatizados na área de biblioteconomia, com a edição da Bibliografia Brasileira de Física. tes relatam experiências em classificação automática. O presente artigo trata somente do primeiro grupo, ou seja, a literatura sobre softwares ou sistemas projetados especificamente para suportar a criação, manutenção e utilização de bases de dados textuais ou sistemas específicos na informatização da catalogação. Exclui, assim, a literatura sobre classificação e indexação automática e, ainda, aquela que aborda aspectos da automação de bibliotecas de maneira geral. Este enfoque justifica-se também pela maior relevância histórica e pelas perspectivas da catalogação automática, seja no aprimoramento dasformas de representação documentária, seja no desenvolvimento de mecanismos para rápido acesso e recuperação da informação. É natural, portanto, maior número de publicações nessa área, o que favoreceu o mapeamento histórico desse processo.

Os principais centros de informação e bibliotecas do mundo podem, hoje, ser conectados por vastas redes on-line. Esse fato faz da catalogação cooperativa uma proposta viável, facilitando, de forma revolucinária, os empréstimos entre bibliotecas e outras atividades de distribuição de recursos informacionais. Pesquisadores acadêmicos podem, por exemplo, usar seus terminais para pesquisar um catálogo representado pela somatória dos acervos de várias bibliotecas, identificar a disponibilidade de um item em cada um desses acervos e, eventualmente, fazer empréstimos de materiais que lhe serão entregues na sua sala ou laboratório. No Brasil, a automação nas principais bibliotecas e centros de documentação já ultrapassou os estágios iniciais de desenvolvimento. Os avanços tecnológicos na área de tratamento da 
informação destacam-se primeiramente nos serviços de catalogação e, depois, na indexação automatizada. Com freqüência, nos projetos de automação de bibliotecas, a primeira demanda se concentra na organização do catálogo e na solução de seus problemas mais freqüentes, como a geração de registros no controle da coleção, sua disponibilização e a flexibilização do seu acesso. Nessa tarefa, o gerente de informação, dentro do novo perfil profissional que delineia-se nessa área, dispõe de três tipos básicos de software:

1. sistemas de gerenciamento de bibliotecas;

2. sistemas de gerenciamento de base de dados bibliográficos;

3. sistemas de gerenciamento de banco de dados.

Os sistemas de gerenciamento de bibliotecas são sistemas de bases de dados com uma finalidade específica, projetados para controlar as atividades essenciais de uma biblioteca. Geralmente, funcionam em computadores de grande porte, destinados a centro de informações maiores, permitindo uma padronização, integração, compatibilidade e intercâmbio de um grande volume de informações. No Brasil, entre os gerenciadores de bibliotecas estão o Virginia Tech Library System (VTLS), utilizado pelo Sistema de Bibliotecas da UFMG, Bibliotecas da Unicamp e pelas Bibliotecas da Universidade de Santa Catarina; o Automated Library Expandable Program (ALEPH), que é um software desenvolvido na The Hebrew University, em Jerusalém, Israel (comercializado pela Ex Libris), implantado na Rede de Bibliotecas da USP e no Sistema de Bibliotecas da Fiocruz; o Sysbibli, desenvolvido pela Contempory, utilizado no Banco do Desenvolvimento de Minas Gerais (BDMG), Universidade de Guarulhos, Agroceres (SP) e Datamec (RJ) e também o Informa Biblioteca Eletrônica, que é utilizado na Assembléia Legislativa do Rio de Janeiro, no Colégio Cristo Rei/ES, no Instituto Cultural Itaú/ SP e na Universidade Metodista/SP.

Gerenciadores de bases de dados bibliográficas, como Endnote, Papirus,
PRO-CITE, Acervum, Reference Manager, MicrolSIS, LightBase, são softwares que rodam em microcomputadores, destinados a uma clientela que inclui não apenas bibliotecários, mas também usuários pessoais, principalmente professores e pesquisadores acadêmicos. São programas que apresentam facilidades e vantagens na sua utilização devido às suas interfaces amigáveis. Alguns vêm com formatos preestabelecidos para atender às necessidades bibliográficas e facilitar a entrada de dados.

Já os Sistemas de Gerenciadores de Banco de Dados, como Access, File Maker e dBASE, são softwares de espectro comercial mais amplo, que suportam o armazenamento de grandes quantidades de informação. Possuem habilidades de recuperação segundo diferentes critérios de cruzamento, o que é uma característica importante para a maior parte das funções bibliotecárias. Apesar de serem considerados opções naturais na manipulação de bases de dados contendo o acervo de uma típica biblioteca brasileira (Sayão et al., 1989), esses gerenciadores apresentam limitações, a exemplo dos sistemas de campos fixos e saídas em forma colunar, que dificultam sua utilização pelo bibliotecário. Comparados com os sistemas de gerenciamento de bibliotecas que permitem informatizar todas as funções de uma biblioteca, tanto os gerenciadores de banco de dados, quanto os gerenciadores de bases de dados bibliográficos têm aplicações restritas em bibliotecas, uma vez que não atendem a diversas demandas de manipulação de informação. Sua adaptação na realização de algumas tarefas pode resultar em um aproveitamento inadequado de algumas funções, ou mesmo comprometer o serviço prestado. Sua utilização, entretanto, é justificável pelo baixo custo e possibilidade de operar em sistemas monousuários.

\section{METODOLOGIA}

A partir da coleta e seleção da biblio- grafia, esse estudo teve um enfoque analítico-descritivo baseado no levantamento quantitativo das seguintes variáveis:

- tipo de documento (artigos em periódicos e trabalhos completos publicados em anais);

- autoria (autor, co-autor, colaborador e tradutor);

• formação profissional dos autores;

- fonte de publicação (título do periódico ou anais);

- local de origem do autor (cidade onde o autor mora ou trabalha);

- data.

\section{ANÁLISE DA LITERATURA SELECIONADA}

Foram pesquisados os catálogos da Biblioteca Etelvina Lima da Escola de Biblioteconomia da UFMG, da Biblioteca do IBICT e as bases de dados online da USP, Unicamp e UFRGS.

Para este estudo, foram considerados somente os softwares citados na literatura brasileira, cujo o universo é menor que a totalidade dos softwares existentes no mercado*. Por isso, sugerese que este levantamento seja complementado com outras fontes como livros, manuais, a rede Internet etc.

Por meio da literatura nacional sobre softwares aplicados à biblioteconomia pôde-se notar uma predominância absoluta de relatos de experiências na utilização do MicroISIS na automação de bibliotecas. Outra parcela significativa dos artigos relata aplicações de softwares comerciais. Há também o caso de sistemas de automação isolados (programas in house). Por desconhecimento da disponibilização de softwares no país, pelo rigor da lei de reserva de mercado na área de informática que predominou até 1993 ou, ainda, pela crença da necessidade de

\footnotetext{
* Alguns softwares utilizados no Brasil não citados na literatura são Acervum, Biblos BookMaster, Docman, EasyBook, EasyDoc, Sistema GIZ Biblioteca, Sistema Thesaurus, SophiA-Biblioteca, Biblioteca Argonauta, YESHÛA, Papyrus, @ULA etc.
} 
softwares sob medida, muitas bibliotecas tentaram desenvolver isoladamente seus próprios programas (ver tabela 1). Essa tendência tende a diminuir devido à dificuldade de os programas in house se comunicarem com outros formatos padronizados ou participarem de redes de intercâmbio e, também, devido à sua desvantagem diante da grande flexibilidade e praticidade proporcionada pelos softwares comerciais.

Notou-se significativo crescimento no número de publicações a partir da crescente adoção do MicroISIS pelas bibliotecas ou centros de documentação. Ao mesmo tempo, o número de textos sobre a aplicabilidade dos outros programas manteve-se pequeno em todo o período (ver tabela 2). Do total de 75 artigos consultados que tratam de catalogação automática e softwares para automação de bibliotecas, centros de documentação ou serviços de informação, 40 tratavamexclusivamente da utilização do software MicroISIS como gerenciador de bases de dados textuais, confirmando que esse ainda é o programa mais utilizado e estudado por bibliotecários no Brasil. Alguns fatores contribuíram para isso. Entre eles, a utilização do MicroISIS pela rede Bibliodata como formato de saída tornando o uma linguagem comum para as bibliotecas do Brasil e da América Latina, bem como a natureza não lucrativa deste programa, desenvolvido pela Unesco e distribuído pelo IBICT desde 1986, e, finalmente, a dificuldade de aquisição de softwares/har$d$ wares funcionalmente mais diversificados no mercado brasileiro.

Observou-se que os textos resultantes de trabalhos apresentados em eventos representaram quase o dobro dos artigos publicados em revistas especiali-
TABELA 1

Classificação dos softwares quanto à disponibilização

\begin{tabular}{lll}
\hline Disponibilização & comercial & in house \\
\hline \multirow{2}{*}{ Softwares } & Aleph, Dobis/Libis, GB, ILS, & Gerbibli, Ainfo, BIB/Dialogo \\
& Sibil, Stairs, Sysbibli, & BIB/Bacth, Saber, SAB, \\
& InMagic Plus Software, & SABi, SAB2, Sistema Calco, \\
& LighBase, MicrolSIS, Ortodocs, & Patplus \\
& Pro-Cite, SRI, VTLS & \\
Total & 14 & 10 \\
\hline
\end{tabular}

TABELA 2

Número de publicações por software

\begin{tabular}{|c|c|c|}
\hline Softwares & Número & $\%$ \\
\hline Ainfo & 02 & 2,66 \\
\hline Aleph & 01 & 1,33 \\
\hline BIB/Bacth & 01 & 1,33 \\
\hline BIB/Dialogo & 02 & 2,66 \\
\hline Dobis/Libis & 03 & 4,00 \\
\hline GB & 01 & 1,33 \\
\hline Gerbibli & 01 & 1,33 \\
\hline InMagic Plus Software & 01 & 1,33 \\
\hline Integrated Library System (ILS) & 02 & 2,66 \\
\hline LighBase & 01 & 1,33 \\
\hline MicroISIS & 40 & 53,33 \\
\hline Ortodocs & 02 & 2,66 \\
\hline Patplus & 01 & 1,33 \\
\hline Pro-Cite & 02 & 2,66 \\
\hline SAB & 01 & 1,33 \\
\hline SAB 2 & 01 & 1,33 \\
\hline Saber & 01 & 1,33 \\
\hline $\mathrm{SABi}$ & 01 & 1,33 \\
\hline Sibil & 01 & 1,33 \\
\hline Sistema Calco & 02 & 2,66 \\
\hline SRI & 01 & 1,33 \\
\hline Stairs & 05 & 6,67 \\
\hline Sysbibli & 02 & 2,66 \\
\hline VTLS & 01 & 1,33 \\
\hline Total & 75 & 100,00 \\
\hline
\end{tabular}


zadas (ver tabela 3). Isso mostra a importância de eventos periódicos como os seminários sobre automação de bibliotecas e centros de documentação que, bienalmente, divulgaram 30,66\% de todos os textos da área (ver tabela 4). Em seguida, aparecem o Congresso Brasileiro de Biblioteconomia e Documentação e o Congresso de Biblioteconomia, Documentação e Ciência da Informação, representando, cada um, $8.0 \%$ do total. Nos outros eventos, as publicações nessa área variaram entre $1,33 \%$ a $5,33 \%$ do total. Seguindo a mesma tendência dos trabalhos completos em anais, os artigos se concentraram em um só periódico, a Revista Ciência da Informação (20\% do total selecionado), secundada pela Revista de Biblioteconomia de Brasília $(10,66 \%)$. Os demais periódicos consultados apresentaram apenas um ou nenhum trabalho.

Geograficamente, notou-se que Brasília destaca-se como maior centro de divulgação de trabalhos sobre softwares para automação de bibliotecas. Juntos, seus dois periódicos, a Revista Ciência da Informação do IBICT e a Revista de Biblioteconomia de Brasília, da UnB, foram responsáveis pela divulgação de quase um terço das publicações (ver tabela 4).

No que diz respeito à procedência dos autores (ver tabela 5), 37,33 \% são de São Paulo, seguidos por $17,33 \%$ do Rio de Janeiro, 16,00\% de Santa Catarina e $10,66 \%$ de Brasília. Os estados de Paraná, Pernambuco, Rio Grande do Sul, Minas Gerais e Espírito Santo apresentaram índices bem menores. Se há maior contribuição dos autores paulistas e depois cariocas, não surpreende, pois a automação em bibliotecas depende diretamente de recursos financeiros significativos. Os autores catarinenses, como Ohira, revelaram-se como importantes lideranças intelectuais na área. Por outro lado, estados ricos, como Minas Gerais e Rio Grande do Sul, demonstraram pouca participação nesta área de estudo.

Em relação à distribuição temporal dos textos (ver tabela 6), notou-se maior coincidência do volume de publicações com os anos de realização dos Seminários sobre Automação de Bibliote-
TABELA 3

\section{Tipos de documentos produzidos}

\begin{tabular}{lcc}
\hline Tipos de publicações & Número & $\%$ \\
\hline Artigos em periódicos & 28 & 37,33 \\
Trabalhos completos em anais & 47 & 62,66 \\
Total & 75 & 99,99 \\
\hline
\end{tabular}

TABELA 4

Fontes das publicações sobre softwares para automação de bibliotecas

\begin{tabular}{lcc}
\hline Periódicos & Número & $\%$ \\
\hline Revista Ciência da Informação & 15 & 20,0 \\
Revista de Biblioteconomia de Brasília & 08 & 10.66 \\
Revista Brasileira de Biblioteconomia e Documentação & 02 & 2,66 \\
Revista Brasileira de Computação & 01 & 1,33 \\
Transinformação & 01 & 1,33 \\
\hline
\end{tabular}

Anais de eventos científicos

Número

$\%$

Seminários sobre Automação de Bibliotecas e

30,66

Centros de Documentação

Congressos Brasileiros de Biblioteconomia e

23

8,0

Documentação

Congressos de Biblioteconomia, Documentação e 06

$06 \quad 8,0$

Ciência da Informação

Painéis de Biblioteconomia de Santa Catarina

Seminários Nacionais de Bibliotecas Universitárias

Congresso Latino-Americano de Biblioteconomia e

Documentação

Encontros Nacionais de Informática e

Documentação Jurídica

Seminários Catarinenses de

Biblioteconomia \& Informação

Total

75

100,0

\section{TABELA 5}

\section{Distribuição geográfica dos autores principais}

\begin{tabular}{llr}
\hline Local & Número & $\%$ \\
\hline São Paulo & 28 & 37,33 \\
Rio de Janeiro & 13 & 17,33 \\
Santa Catarina & 12 & 16,00 \\
Brasília & 08 & 10,67 \\
Paraná & 04 & 5,33 \\
Pernambuco & 04 & 5,33 \\
Rio Grande do Sul & 03 & 4,00 \\
Minas Gerais & 02 & 2,67 \\
Espírito Santo & 01 & 1,33 \\
Total & 75 & 100,00 \\
\hline
\end{tabular}


cas e Centros de Informação $(1989,1992,1994,1997)$. O ano mais produtivo, considerando a data dos trabalhos selecionados, foi o de 1994. Esse fato deveu-se à realização de três eventos no mesmo ano: o Seminário sobre Automação de Bibliotecas e Centros de Informação, o Seminário Nacional de Bibliotecas Universitárias e o Congresso Latino-Americano de Biblioteconomia e Documentação. Pôde-se observar ainda que, à exceção do ano de 1985 (com seis publicações), foi a partir da consolidação do MicrolSIS no Brasil (cujo primeiro artigo data de 1988) que surgiu um número maior e mais consistente de publicações.

Os autores brasileiros que publicaram dois ou mais trabalhos nessa área (ver tabela 7) foram Ohira, Gildenir SANTOS, McCarthy, Salvato, Sell, Cruz, Pimentel e Souza. Dos 131 autores levantados, somente 29 publicaram individualmente. Em contraposição, 46 das 75 publicações resultaram de coautoria, o que sugere um típico envolvimento e consolidação de equipes de trabalho nesta área.

Quanto à formação profissional dos autores (ver tabela 8), notou-se que a maioria é de bibliotecários $(61,07 \%)$, seguidos por analistas de sistemas $(12,21 \%)$ e professores universitários $(11,45 \%)$. Estes dados tornam-se preocupantes, se levarmos em conta a expectativa de que a universidade seja o principal pólo de pesquisa e geração de conhecimento no país. Se o estudo e utilização desses softwares ainda não atraiu a devida atenção da comunidade bibliotecária acadêmica, notase, por outro lado, a formação de grupos de estudo entre os bibliotecários. Confirmando este quadro está o grande número de relatos de experiência publicados.
TABELA 6

Número de publicações por ano

\begin{tabular}{rrr|rrr|rrr}
\hline Ano & Número & $\%$ & Ano & Número & $\%$ & Ano & Número & $\%$ \\
\hline 1998 & 2 & 2,66 & 1992 & 11 & 14,66 & 1986 & 2 & 2,66 \\
1997 & 10 & 13,33 & 1991 & 8 & 10,66 & 1985 & 6 & 8,00 \\
1996 & 0 & 0,00 & 1990 & 2 & 2,66 & 1984 & 0 & 0,00 \\
1995 & 0 & 0,00 & 1989 & 6 & 8,00 & 1983 & 0 & 0,00 \\
1994 & 19 & 25,33 & 1988 & 2 & 2,66 & 1982 & 1 & 1,33 \\
\hline
\end{tabular}

TABELA 7

Publicações por autor

Autor

Número

$\%$

OHIRA, Maria Lourdes B.

6,66

SANTOS, Gildenir

5,33

McCARTHY, Cavan Michael

4,00

SALVATO, Gilberto José

4,00

SELL, Maria Aparecida

4,00

CRUZ, Angela M. V. da

2,66

PIMENTEL, Carlos Eduardo

2,66

SOUZA, Irene Josefa

2,66

Demais autores

1,33

\section{TABELA 8}

\section{Formação profissional dos autores}

\begin{tabular}{lcr}
\hline Formação Profissional & Número & $\%$ \\
\hline Bibliotecários & 80 & 61,07 \\
Analistas de sistemas & 16 & 12,21 \\
Professores universitários & 15 & 11,45 \\
Alunos & 09 & 6,87 \\
Chefias administrativas & 05 & 3,81 \\
Consultores empresariais & 04 & 3,07 \\
Assessor de informática & 02 & 1,52 \\
Total & 131 & 100,00 \\
\hline
\end{tabular}

\section{APRESENTAÇÃO DOS SOFTWARES CITADOS NA LITERATURA}

Esta seção descreve, em ordem alfabética e a partir da bibliografia consul- 
tada, as características básicas e locais de aplicação de 24 softwares. Ao mesmo tempo em que visa a reunir informações sobre programas virtualmente desconhecidos da maioria dos bibliotecários e professores de biblioteconomia, pretende servir como ponto de partida para comparações e eventuais aquisições.

\section{Ainfo}

Ainfo é uma ferramenta para gerenciamento de bases de dados documentais e processos bibliográficos, desenvolvida pelo Centro Nacional de Pesquisa Tecnológica em Informática para Agricultura (CNPTIA) em Campinas, São Paulo, para ser utilizada no ambiente de software NTIA, criado na própria Embrapa. Durante o V Seminário de Automação em Bibliotecas e Centro de Documentação, Lenk (1994) apresentou sucintamente esse aplicativo, explicando como se dá o gerenciamento local da informação técnicocientífica nas unidades de pesquisa dessa empresa, distribuídas por todo o território nacional. No VI Seminário de Automação em Bibliotecas e Centro de Documentação, Oliveira e Antures (1997) apresentaram o Ainfo mais detalhadamente, descrevendo sua estrutura, seus módulos básicos e os relacionamentos entre suas bases de dados.

\section{Aleph (Automated Library Expandable Program)}

O software Aleph é um gerenciador de bibliotecas, desenvolvido na The Hebrew University, em Jerusalém, Israel. Comercializado no Brasil pela Ex Libris, está sendo utilizado por instituições como a USP e a Fundação Osvaldo Cruz (Fiocruz). Durante o VI Seminário sobre Automação de Bibliotecas e Centros de Documentação, Moraes (1997), relatou a experiência de automação do Sistema de Bibliotecas da Fiocruz, particularmente o Programa de Controle de Qualidade Total na Biblioteca de Manguinhos. Abordou também a mudança na indexação dos documentos para cooperação com as bases Lilacs e Secs.

\section{BIB/Dialogo e BIB/Batch}

Desenvolvidos por Jaime Robredo no
Departamento de Biblioteconomia da Universidade de Brasília e com o apoio do Centro de Processamento de Dados daquela universidade, o BIB/Dialogo e o BIB/Batch são sistemas escritos em linguagem Cobol standard, para computadores Burroughs B6700 e terminais Skopus TVA 800, de fabricação nacional. Enquanto o primeiro é um sistema em linha para armazenagem e recuperação de dados bibliográficos, o segundo é um sistema completo de processamento da informação documentária em lotes. O próprio Robredo (1982), no artigo Dois novos sistemas com computadores para o processamento completo da informação documentária, sugeriu que esses seriam os primeiros sistemas de informática documentária desenvolvidos e utilizados para fins de ensino e pesquisa em uma universidade de um país em desenvolvimento. Moreia (1991) relatou sua experiência utilizando o BIB/Dialogo durante o planejamento e criação do banco de dados Pró-música sobre suportes de informação musical, projeto que foi o trabalho final do autor na disciplina Tópicos Especiais em Documentação e Ciência da Informação, do Curso de Mestrado em Biblioteconomia e Documentação da Universidade de Brasília.

\section{Dobis/Libis}

Em 1991, dois trabalhos foram apresentados no XVI Congresso Brasileiro de Biblioteconomia e Documentação relatando as experiências de automação de acervos da Secretaria Municipal de Cultura de São Paulo utilizando o Dobis/Libis. No primeiro trabalho, Hueso, Mattes e Bertonazzi descrevem sua utilização na implantação do sistema Fast-cat, criado para atender às funções de pré-catalogação e agilizar o processamento técnico, após a incorporação do acervo do Departamento de Bibliotecas Infanto-Juvenis. No segundo trabalho, Mattes aponta as dificuldades na implantação desse software pelo Departamento de Bibliotecas Públicas e os problemas decorrentes da falta de critérios técnicos básicos (inexistência de suporte, treinamento de usuários, levantamento das necessidades dos usuários etc). Relata, também, o processo de automação do acervo bibliográfico, documen- tal e musical da Secretaria de Cultura do Município de São Paulo.

A experiência de informatização da rede da Secretaria Municipal de Cultura de São Paulo (SMC) foi ainda relatada por Melo, Brandão e Santos (1994) no Seminário sobre Automação de Bibliotecas e Centros de Documentação. As autoras descrevem esse gerenciador de bibliotecas de origem belga-alemã que pode atuar em rede. O Dobis/Libis é conversacional, multilíngüe, compatível com o formato Marc, com descrição bibliográfica para todos os tipos de materiais e emissão de relatórios e estatísticas. Suas funções de pesquisa, catalogação, resumos, circulação, aquisição, controle de periódicos, correio eletrônico e saídas impressas (como emissão de etiquetas de código de barras) podem ser integradas. Sua rede no Brasil contava, na data da publicação do artigo, com a participação de 79 bibliotecas. Contava também com serviços periódicos de manutenção e cursos e atualização.

\section{GB (Gestor de Bibliotecas)}

Brunhera (1992) relatou como, juntamente com analistas de sistemas, criou esse software para gerenciamento de bibliotecas e centros de informação e documentação. Distribuído pela Biblioinfo S/C Ltda., o GB foi desenvolvido para atender especialmente às bibliotecas especializadas em direito. Entre as principais características deste software, a autora destaca a catalogação de uma grande diversidade de materiais (inclusive jurisprudência), a recuperação simplificada por meio de várias entradas, o fácil controle de empréstimos, a possibilidade de impressão de listagens, a manutenção de estatísticas atualizadas e o help online.

\section{GERBIBLI(Gerenciador de}

Bibliotecas)

O sistema Gerbibli foi descrito por Santos, Truzzi e Ferroni em 1994. Surgido de um trabalho integrado entre analistas e bibliotecários, este software foi desenvolvido para cadastra- 
mento, controle e recuperação da informação de materiais bibliográficos. Criado para atender às necessidades da biblioteca especializada da BPS (uma empresa de projetos e integração de sistemas nas área de telecomunicações, automação e informática) o Gerbibli possui quatro conjuntos bibliográficos (livro, manual, periódico e catálogo) e cinco comandos (cadastro, etiqueta, movimento, remover e pendência). Os nomes dos comandos são auto-explicativos, não havendo dificuldade na uso de suas funções.

\section{ILS (Integrated Library System)}

O ILS, desenvolvido pelo Centro de Processamento de Informações Científicas da Comunidade Européia, Itália, serve à automatização de todos os serviços de uma biblioteca. Sousa, Bellizia e Guedes (1989) relataram a experiência da Divisão de Informação e Documentação Científica (DIDC) do Instituto de Pesquisas Energéticas e Nucleares (Ipen) com esse software na automação do seu sistema e restruturação do catálogo de assunto. Mais tarde, no V Seminário sobre Automação de Bibliotecas e Centros de Documentação, Igami (1994) expôs os motivos que levaram as bibliotecas do Ipen a trocar de software, relatando como se deu a migração de dados para sistemas alternativos. Lourenço e Cavalieri (1994) também citaram o ILS como uma alternativa de software estrangeiro disponível no mercado nacional. Deve-se observar, porém, que não há na literatura uma descrição mais detaIhada sobre o mesmo.

\section{InMagic Plus}

O InMagic Plus foi criado nos EUA na década de 80 , tendo sido utilizado em mais de 50 países. No Brasil, foi estudado em um projeto-piloto na USP, apresentado por Duckworth (1994) no V Seminário de Automação em Bibliotecas e Centros de Documentação. Com esse software, criou-se a base de dados Projetos, que facilitava 0 acesso aos relatórios dos projetos de pesquisa da Escola do Futuro, em um programa ligado à Pró-Reitoria de Pesquisa da USP, otimizando suas informações e gerenciamento.

\section{LightBase}

Segundo Pimentel e Pimentel (1994), o LightBase é um software destinado ao gerenciamento de bases de dados textuais, que possibilita o armazenamento e recuperação de informações por meio de pesquisas por qualquer dado, ou seja, palavra, valor, data etc., sem necessidade de marcação prévia. Os autores descreveram a utilização desse softwareno desenvolvimento inovador de uma base de dados de usuários, destacando a importância da mesma para melhorar o relacionamento dos bibiotecários com o público. Como principais características do LightBase, apontaram sua interface amigável, a facilidade de pesquisa e a enorme capacidade em relação ao do tamanho das bases de dados e extensão dos registros.

\section{MicrolSIS}

No final de 1985, adaptando o Sistema ISIS que operava em computadores de grande porte, a Unesco lançou o MicroISIS, especialmente em países em desenvolvimento, para gerir bases de dados documentais em microcomputadores IBM-PC e compatíveis. Este programa permite construir e gerenciar bases de dados estruturadas e nãonuméricas, derivadas de coleções de objetos homogêneos, constituídas principalmente de textos descritivos. A popularização do MicrolSIS no Brasil, a partir do início de sua distribuição pela Unesco, em 1986, deu-se nas mais diversas áreas do conhecimento. Sua aplicação vai da catalogação automatizada de fotografias até a criação de guias de fontes de informação. O primeiro artigo sobre esse programa, Implantação do MicroISIS no Senac, relata sua experiência pioneira no país. Nele, Cruz e Paes Leme (1988) descrevem o programa, bem como etapas de planejamento e definição das bases de dados do Senac, a definição de padrões para entrada de dados e a formatação de relatórios e pesquisa. Fazem também uma análise dos pontos positivos e negativos de sua estrutura. Posteriormente, Miki (1989) publica MicroISIS: uma ferramenta para o gerenciamento de bases de dados bibliográficas, que é, ainda hoje, uma referência básica na literatura sobre esse programa. Nesse artigo, o autor descreve as características funcionais do
Micro-ISIS e recursos disponíveis, bem como suas limitações e perspectivas no Brasil. A publicação mais recente sobre o MicroISIS no Brasil foi apresentado por Lima e Mendonça (1998), em que sumariamos, historicamente e por áreas de aplicação, outros 37 artigos sobre esse programa, publicados desde 1988. Mencionamos também as mais recentes versões do programa e endereços eletrônicos para demonstração, aquisição e fórum de discussão internacional. Atualmente, no Brasil, as informações sobre o MicroISIS concentram-se nos sites da Bireme cujo o endereço eletrônico é http//: www.bireme.br e da Contempory www.contempory.com).

(http//:

\section{Ortodocs}

O Ortodocs, fruto da tecnologia brasileira de microinformática, permite desenvolver várias funções da biblioteca, incluindo a catalogação, reconversão de catálogos e a geração de bases de dados de autoridade, reunindo autores pessoais/coletivos e cabeçalhos de assunto. Zanaga (1994) descreveu a mudança dos procedimentos adotados na catalogação automatizada do Sistema de Bibliotecas da Unicamp e justifica a adoção do Ortodocs por agilizar a catalogação e permitir que todos os procedimentos sejam efetuados online, desde a pesquisa do acervo até a inserção de um novo registro ou aproveitamento de registros já existentes na Base Bibliodata/Calco. Almeida et al. (1994) relataram o projeto de automação da Rede de Bibliotecas da Unesp utilizando esse software, destacando sua característica de permitir a importação de registros bibliográficos de qualquer formato e convertê-los em formato Marc.

\section{Patplus}

O Patplus é um sistema para recuperação de informação em bancos de dados organizados na forma de texto completos, implementado em linguagem Pascal para microcomputadores de 16 bits. Utiliza um sistema operacional do tipo DOS e requer uma memó- 
ria interna mínima de $256 \mathrm{~KB}$ e discos flexível. Zivianni (1990) descreveu este programa, suas características e módulos principais: o módulo de conversão, o módulo que permite a construção de um índice invertido e o módulo de consulta. Naquela época, o Patplus estava sendo utilizado experimentalmente em alguns departamentos da UFMG, instituição na qual foi desenvolvido com a coordenação do autor anteriormente citado.

\section{Pro-Cite}

Desenvolvido em 1983 por um professor de biblioteconomia da Universidade de Michigan, a primeira versão do Pro-Cite chamava-se Personal Bibliographic System e funcionava em microcomputadores Apple. No Brasil, este software foi estudado por McCarthy $(1989,1991)$. No primeiro artigo, o autor apresentou seu sistema de definição de dados bibliográficos. Por ser um software destinado ao gerenciamento de referências bibliográficas, o ProCite destaca-se por oferecer formatos prontos para materiais bibliográficos e audiovisuais. Bastante amigável, dispõe de menus ou listas de comandos em cada etapa, sendo muito apropriado às bases de dados pequenas. $\mathrm{Na}$ entrada de dados, o sistema permite, em todos campos, um número ilimitado de qualquer tipo de caracteres. Dentre as principais vantagens desse sistema, podemos destacar a facilidade na entrada de dados, a produção de bibliografias e referências bibliográficas, a geração de índices e a economia de memória. Como desvantagens, o autor destacou o custo (US\$395.00), a pouca difusão no país e a destinação ao usuário pessoal com limitações nas operações em bibliotecas. Um outro problema é o fato de o formato de saída do sistema estar de acordo a normalização bibliográfica norte-americana, e não da ABNT.

No segundo artigo sobre o Pro-Cite, McCarthy (1991) relata a utilização desse software na criação de uma base de dados para publicações locais em Pernambuco. Esse projeto tinha, como um dos seus principais objetivos, registrar publicações especializadas, a "literatura cinza"* e outras publicações não submetidas ao controle bibliográ- fico. A entrada catalográfica semelhante ao AACR2 e o formato final compatível com outros formatos bibliográficos, como Marc e Calco, foram apontados como pontos bastante positivos. Apesar de o software não possuir limites de caracteres nos campos, um dos problemas encontrados foi a inclusão de letras acentuadas, cuja solução foi sua representação com códigos de três dígitos.

SAB (Sistema de Automação de Bibliotecas)

Desenvolvido na biblioteca técnica da BSM - Sistema e Métodos S.A, o SAB tinha como objetivo automatizar todos os serviços oferecidos por esta empresa. Medina e Albuquerque (1986) apresentam esse software como sendo destinado basicamente à recuperação e armazenamento de informações bibliográficas através de funções on-line.

\section{SAB2 (Sistema de Automação de Bibliotecas 2)}

Resultado de um convênio entre a Fundação Universidade do Rio Grande-RS (FURG), a Fundação Getúlio Vargas e a IBM do Brasil, o SAB2 foi criado com o objetivo de desenvolver e implantar um sistema de automação de bibliotecas que permitisse a integração desse sistema ao processo de catalogação cooperativa baseado no padrão nacional de intercâmbio de dados bibliográficos.

Becalli et al. (1994) relataram a experiência de utilização desse software na informatização do Sistema Integrado de Bibliotecas da Universidade Federal do Espírito Santo (SIB/Ufes). Os autores descreveram o SAB2 como um aplicativo que permite a implantação imediata de serviços e produtos, tais como cadastro de usuários, controle de circulação de acervo, pesquisa bibliográ-

"O autor chama de "literatura cinza" os documentos periféricos de menor divulgação. fica, além de permitir o acesso on-line.

\section{Saber}

O Saber foi desenvolvido em forma interativa, para computadores de grande porte, do tipo IBM 3032. Sá, Donnard e Dantas Filho (1985) descreveram esse sistema projetado com objetivo de processar, arquivar e recuperar, automaticamente por meio de disco magnético, referências bibliográficas de interesse dos usuários da Biblioteca Central do IBGE. Além da recuperação em linha, com utilização da lógica booleana, e da emissão automática do boletim bibliográfico, o sistema permite a emissão de listagens por qualquer um dos campos recuperáveis, bem como listagens de estatística de uso.

SABi (Sistema de Automação de Bibliotecas da UFRGS)

SILVA et al. (1994) descreveram o SABi como um sistema integrado, desenvolvido pela UFRGS, que prevê automação de todas as funções de bibliotecas universitárias. Elaborado com base no CCAA2, esse software foi implantado em 1989 para organizar monografias e publicações seriadas, podendo também incluir materiais especiais. Utiliza o MicrolSIS para geração de alguns módulos, sendo compatível com o Formato IBICT. O SABi foi disponibilizado à comunidade pela Internet.

\section{Sibil (Sistema de Informações Bibliográficas)}

Desenvolvido pela Scientia e pela Companhia de Tecnologia Industrial, o Sibil é composto de seis subsistemas: entrada e crítica de dados; monitoração de acesso e uso; pesquisa bibliográfica em modo conversacional; disseminação seletiva de informações, apoio documental; controle de empréstimo e reservas. Oliveira e Araújo (1985) relataram a experiência com esse software na implantação do sistema de automação do acervo e serviços da rede de bibliotecas do Serviço Federal de Processamento de Dados (Serpro). Além de permitir a organização dos catálogos individuais de cada biblioteca, por autor, título e assunto, o sistema emite etiquetas e um boletim bibliográfico, que funciona como catálogo coletivo de todo o acervo da rede, evitando 
aquisições desnecessárias e duplicação do acervo.

\section{Sistema Calco}

O Sistema Calco foi desenvolvido pela Fundação Getúlio Vargas (FGV) a partir do Sistema Marc, da Library of Congress, para automatizar os processos de catalogação cooperativa, permitindo o intercâmbio via rede Bibliodata. Aronovich, Alves e Dias (1985) descreveram o processo de automação na Biblioteca Nacional com a adoção do formato Calco e sua integração ao sistema Bibliodata/Calco em 1982. Decourt (1987), no artigo O sistema CALCO e a Rede Bibliodata, descreveu esse sistema, seus objetivos e produtos oferecidos, frisando que sua grande vantagem é a cooperação, permitindo que várias bibliotecas utilizem simultaneamente e contribuam com a entrada de dados no mesmo arquivo central de dados. Implantado como banco de dados, esse sistema permitia consultas off-line.

SRI (Sistema de Armazenamento e Recuperação de Informações)

O SRI é um banco dados de informações textuais criado pela Pantheon Informática, empresa nacional sediada em Brasília. Schaurer (1992) relatou a utilização do SRI no processo de automação no Centro de Documentação da Price Waterhouse, empresa prestadora de serviços na área de auditoria, consultoria, planejamento, informação e controle empresarial. O autor ressaltou que a montagem e gerência de bancos de dados criados com o SRI pode ser feita por qualquer bibliotecário ou auxiliar de biblioteca, com conhecimento mínimo de informática. Contribuem para isto os menus em português e o recurso da lógica booleana, que permite uma pesquisa bastante rápida.

\section{Stairs (Storage and Information Retrieval System)}

Segundo Graeff e Lima (1985), esse software da IBM consiste de um conjunto de programas que permite ao usuário, através de um terminal, dialogar diretamente com o computador. Além de sua utilização no Prodasen (Centro de Processamento de Dados do Senado Federal), descrita anteriormente pelos autores, há diversas experiências com este software no Brasil. Miashiro (1985) descreveu a utilização desse software na implementação do acesso on-line do Sistema de Informação Técnico-Científico (Sitce) da Embrapa. Teixeira e Gomes (1986) apresentaram a automação dos serviços bibliográficos da Rede Ferroviária Federal S.A., enquanto Nunes et al. (1991) trataram da automatização das bibliotecas da Universidade do Rio Grande, ressaltando sua importância na aceleração deste processo. Finalmente, Soler (1992) descreveu a experiência com o Stairs no Centro de Informação Tecnológica da Metal Leve S/ A (CIT), explicando que sua escolha se deu por permitir o trabalho com operadores lógicos (lógica booleana), por rodar em mainframe e pela sua potência na recuperação da informação.

\section{Sysbibli}

Lourenço e Cavalieri (1994) apresentaram o gerenciador de bibliotecas Sysbibli como um software com aplicativos completos para a automação de arquivos, bibliotecas e centros de informação. Esse sistema engloba as rotinas para a mecanização dos processos técnicos, atendimento ao usuário e rotinas administrativas. Desenvolvido no Brasil pela Contempory - Consultoria Empresarial, era comercializado em três versões: uma para bibliotecas públicas, outra para bibliotecas universitárias e escolares e uma terceira para bibliotecas especializadas. Suas principais funções são o gerenciamento de acervo a um custo reduzido, a racionalização e organização dos processos técnicos, além da promoção da disseminação da informação em vários níveis. Anteriormente, Cruz e Kelmer (1990) haviam descrito a aplicação do Sisper - Sistema de Controle de Automático de Periódicos (um dos cinco módulos do Sysbibli) - no Senac.

\section{VTLS (Virginia Tech Library System)}

O VTLS é um gerenciador de bibliotecas que oferece todas as funções de catalogação e atende a outras necessidades de automação das atividades diárias de unidades de informação. Souza, Catarino, Santos (1997), no artigo Metadados: catalogando dados na Internet, citam a utilização desse software pela Fundação Getúlio Vargas na modernização tecnológica da rede Bibliodata. Nessa mudança, a migração da base de dados para uma plataforma cliente-servidor possibilitou a disponibilização da base de dados em cdrom, a consulta on-line via Internet, a implantação de um novo sistema de entrada de dados e a utilização de sistemas locais de automação compatíveis com os padrões internacionais no formato Usmarc.

\section{CONCLUSÃO}

Entre os fatores que influíram no crescente aumento de publicações que tratam de softwares para bibliotecas e centros de documentação no Brasil, destacam-se (1) a popularização do MicroISIS no país, a partir de 1986, e sua utilização pela rede Bibliodata como formato padronizado de saída, (2) a nova regulamentação do mercado brasileiro de informática, que facilitou a importação de softwares comerciais, (3) o surgimento de sistemas nacionais isolados (in house) e (4) a organização periódica de eventos nacionais.

Brasília destaca-se como o maior centro de divulgação de trabalhos, ancorada por dois periódicos, Ciência da Informação e Revista de Biblioteconomia de Brasília. Quanto a procedência dos autores, São Paulo, Rio de Janeiro, Santa Catarina e Brasília lideram com um maior número de autores, respectivamente. A maioria dos autores é constituída de bibliotecários, ao passo que os professores universitários ainda não se debruçaram significativamente para estudar os avanços tecnológicos da área. Este dado ainda revela que as publicações, predominantemente relatos de experiência, são mais descritivas, retratando mais a prática do que a reflexão teórica. A predominância de co-autoria nos trabalhos - 46 das 75 publicações - mostra uma tendência de formação de grupos de estudo entre profissionais da área, sugerindo ainda o envolvimento, integração e consolidação de equipes de trabalho com perfis diversificados.

A tendência inicial de se utilizar sistemas automatizados isolados (in house), 
ou programas comerciais adaptados à biblioteca, parece dar lugar ao desenvolvimento e aperfeiçoamento de novos softwares que correspondem mais às expectativas dos profissionais e usuários, contribuindo para o aumento do número de bibliotecas e centros de informação automatizados.

Com a chegada de sistemas gerenciadores de bibliotecas, como o ALEPH e o VTLS, abrem-se as portas para os serviços de cooperação com a utilização de um formato bibliográfico único, permitindo a padronização das informações, a compatibilização de dados e o acesso on-line. O número de fornecedores de softwares apresentou um aumento significativo, trazendo mais opções ao mercado brasileiro, o que dá mais liberdade ao profissional da área na escolha de programas para automação de bibliotecas e centros de documentação.

\section{BIBLIOGRAFIA SELECIONADA}

1. ALMEIDA, Glaura Maria Oliveira Barbosa et al. Projeto de automação da rede de bibliotecas da UNESP. In: SEMINÁRIO SOBRE AUTOMAÇÃO DE BIBLIOTECAS E CENTROS DE DOCUMENTAÇÃO, 5, 1994, São José dos Campos. Anais... São José dos Campos : UNIVAP, 1994. p.141146

2. ARAÚJO, Francisca Helena Martins, et al. O tratamento técnico e preservação de fotografias: o projeto de preservação e conservação do acervo fotográfico da Biblioteca Nacional (PROFOTO). In: CONGRESSO LATINO-AMERICANO DE BIBLIOTECONOMIA E DOCUMENTAÇÃO, 2,
1994, Belo Horizonte. Anais... Belo Horizonte: ABMG, 1994. p. 575-97

3. ARONOVICH, Giselda Brasil, ALVES, Marília Amaral Mendes, DIAS, Suely. Processos de automação na Biblioteca Nacional. R. Bibliotecon. Brasília, v.13, n.2, p.195-204, jul./dez.1985.

4. AZEVÊDO, Aline da Rin Paranho de. Tratamento da informação em coleções científicas de zoologia. Ciência da Informação, Brasília, v.21, n.1, p. 59-61, jan./abr. 1992.

5. BACK, Maria Helena de Carlos, SALVATO, Gilberto José. Indexação informatizada de documentos da biblioteca do TRE de Santa Catarina utilizando-se o software MicroISIS. In: : CONGRESSO DE BIBLIOTECONOMIA, DOCUMENTACÃO E CIÊNCIA DA INFORMAÇÃO, 1992, São Paulo. Anais... São Paulo: APB, 1992. p. 297303.

6. BECALLI, Angela Maria et al. Informatização do Sistema Integrado de Bibliotecas da Universidade Federal do Espirito Santo: relato de experiência. In: SEMINÁRIO SOBRE AUTOMAÇÃO DE BIBLIOTECAS E CENTROS DE DOCUMENTAÇÃO, 5, 1994, São José dos Campos. Anais.... São José dos Campos : UNIVAP, 1994 p.57-61.

7. BRANDO, Helena. MEM: uma base de dados bibliográfica sobre a produção técnico-cientifica do CNEM. CONGRESSO BRASILEIRO DE BIBLIOTECONOMIA E DOCUMENTAÇÃO, 16, Salvador, 1991. Anais... Salvador: APBEB, 1991, v.2, p.949-967.

8. BRITO, Claúdio J. Gerenciador de bases de imagens usando o MicrolsIS. Ci. Inf., Brasília, v 22,n.3, p.248-52, set./out. 1993.

9. BRUNHERA, Maria Inês Prado. GB- Gestor de bibliotecas. In: CONGRESSO DE BIBLIOTECONOMIA E DOCUMENTAÇÃO E CIÊNCIA DA INFORMAÇÃO, 1992 , São Paulo. Anais... São Paulo: APB, 1992, p. 334-336.

10. CASTRO, Regina Célia F., PACKER, Abel Laerte, CASTRO, Elenice de. Projeto LILACS/CD-ROM - Literatura Latino Americana e do Caribe em Ciência da Saúde em disco compacto. Revista Brasileira de Biblioteconomia e Documentação, São Paulo, v.22, n.1/2, p.105114, jan./jun. 1989

11. CRUZ, Angela Maria Viana da, KELMER, Henrique. Controle automático de periódicos no SENAC. Ci. Inf. Brasília, v.19, n.1, p.86-89, jan./jun. 1990.

12. CRUZ, Ângela Maria Viana, LEME, Neli Menezes Paes. Implantação do MicroISIS no SENAC. Ci. Inf., Brasília, v.17, n.2 ,p.131-39, jul./dez. 1988.

13. DECOURT, Eugênio. O sistema Calco e a Rede Bibliodata. R.Bibliotecon. Brasília, v.15, n.1, p.79-84, Jan./Jun. 1987.

14. DUCKWORTH, Ana Maria. "InMagic Plus Software: utilização em projeto piloto na Escola do Futuro, Pró-Reitoria de Pesquisa/USP. In: SEMINÁRIO SOBRE AUTOMAÇÃO DE BIBLIOTECAS E CENTROS
DE DOCUMENTAÇÃO, 5, 1994, São José dos Campos. Anais...São José dos Campos : UNIVAP, 1994. p.189.

15. FERRAZ, Terezune Arantes, SOUZA, Irene Josefa, ZAMPERETTI, Marina. A geração da base de dados do Instituto de Pesquisas Energéticas e Nucleares: problemas e perspectivas. R. Bibliotecon. Brasília, v.13, n.1, p.103-114, Jan./jun. 1985.

16. FERREIRA, Sueli Mara Soares Pinto et al. "FUTURA" : uma base de dados sobre a biblioteca do futuro. Ci. Inf., Brasília, v. 26, n.2, p.218-220, maio/ago. 1997.

17. FIERLI, Agalaé de Lima, CATARINO, Maria Elisabete, OKANO, Yoshihiro. EFIS: Base de dados bibliográfico dos trabalhos acadêmicos do Centro de Educação Física e Desportos da Universidade Estadual de Londrina. In: SEMINÁRIO SOBRE AUTOMAÇÃO DE BIBLIOTECAS E CENTROS DE DOCUMENTAČ̃̃O, 6, 1997, Águas de Lindóia. Anais... São José dos Campos: INPE, 1997. p.219-223.

18. FRANCISCO, Maria Helena di et al. BBiBanco Bibliográfico do Instituto de Física e Química de Sâo Carlos. In: SEMINÁRIO SOBRE AUTOMAÇÃO DE BIBLIOTECAS E CENTROS DE DOCUMENTAÇÃO, 5, 1994, São José dos Campos. Anais.... São José dos Campos : UNIVAP, 1994. p. 114-123.

19. FUNARO, Vânia Martins Bueno de Oliveira, FERREIRA,Marta Nosé. Aplicação do MicroISIS, utilizando a metodologia LILACS para compilação da Bibliografia Brasileira de Odontologia. In: SEMINÁRIO SOBRE AUTOMAÇÃO DE BIBLIOTECAS E CENTROS DE DOCUMENTAÇÃO, 5, 1994, São José dos Campos. Anais.... São José dos Campos : UNIVAP,1994 p. 124-130

20. GRAEFF, Candida Maria Piragibe, LIMA, Yone Maria Correa de. As bases de dados da biblioteca do Senado Federal e a sua operação por uma rede de bibliotecas. Rev. Bibliotecon. Brasília, v.13, n.2 p.169-78, jul./dez. 1985.

21. HUESO, Lilia Maria Faccio, MATTES, Raquel N., BERTONAZZI, Sônia Regina Céu. Sistema Fastcat: a pré-catalogação de 300.000 exemplares. In: CONGRESSO BRASILEIRO DE BIBLIOTECONOMIA E DOCUMENTAÇÃO, 16, Salvador. Anais.. Salvador: APBEB, 1991 p. 1064-1076.

22. IGAMI, Mery P. Zamudio. O Processo de migração do acervo bibliográfico da $\mathrm{Bi}$ blioteca do IPEN. In: SEMINÁRIO SOBRE AUTOMAÇÃO DE BIBLIOTECAS E CENTROS DE DOCUMENTAÇÃO, 5, 1994, São José dos Campos. Anais... São José dos Campos : UNIVAP, 1994. p. 147-149.

23. LENK, Leila Maria. Aplicativo AINFO. In: SEMINÁRIO SOBRE AUTOMAÇÃO DE BIBLIOTECAS E CENTROS DE DOCUMENTAÇÃO, 5, 1994, São José dos Campos. Anais... São José dos Campos : UNI- 
VAP, 1994. p.190.

24. LIMA, Gercina Ângela Borém, MENDONÇA, Andraine M.. A Utilização do MicrolSIS no Brasil. Perspectiva em Ciência da Informação, Belo Horizonte, v.3, n.1, p.81-95, jul./dez. 1998.

25. LOBO, Maria de Fátima Diniz, BARCELLOS, Silvia de Oliveira. Guias de fontes de informação: metodologia para geração e automação. Ciência da Informação, v.21, n.1, p.75-81, jan./abr. 1992.

26. LOPES, Roseane R. Velho. Automação para otimizar o tratamento, acesso e fluxo de documentos e informações multilíngues em centros de pesquisa e desenvolvimento. In: SEMINÁRIO SOBRE AUTOMAÇÃO DE BIBLIOTECAS E CENTROS DE DOCUMENTAÇÃO, 3, 1989, Águas de Lindóia. Anais ... São José dos Campos: INPE, 1989. p. 13-6.

27. LOURENÇO, Cintia de Azevedo, CAVALIERI, Ana Beatriz C. Sysbibli: um software aplicativo para automação de bibliotecas. In: SEMINÁRIO SOBRE AUTOMAÇÃO DE BIBLIOTECAS E CENTROS DE DOCUMENTAÇÃO, 5, 1994, São José dos Campos. Anais...São José dos Campos : UNIVAP,1994 p.183-188.

28. MARCHIORI, Patricia Zeni. A Comissão de articulação de informações do núcleo interdisciplinar de meio ambiente e desenvolvimento da Universidade Federal do Paraná. In: SEMINÁRIO SOBRE AUTOMAÇÃO DE BIBLIOTECAS E CENTROS DE DOCUMENTAÇÃO, 5, 1994, São José dos Campos. Anais... São José dos Campos : UNIVAP, 1994. p. 131-133.

29. MARCONDES, Carlos Henrique. Base de dados de teses e produção acadêmica gerada através da conversão de dados entre aplicativo MicroISIS e o sistema de coleta de dados sobre pós-graduação da CAPES. In: SEMINÁRIO NACIONAL DE BIBLIOTECAS UNIVERSITÁRIAS, 8, 1994, Campinas. Anais ... Campinas: UNICAMP, 1994. p. 339-47.

30. MARTINS, Mariza. SANTOS, Nélcia F. B. Planejamento e desenvolvimento da base dados Legi usando o Micro-ISIS. Ci. Inf., v.21, n.1, p.142-143, jan/abr.1992.

31. MATTES, Raquel Naschenveng. Automação do acervo bibliográfico, documental \& musical da Secretaria de Cultura do Município de São Paulo. In: CONGRESSO BRASILEIRO DE BIBLIOTECONOMIA E DOCUMENTAÇÃO, 16, Salvador. Anais... Salvador: APBEB, 1991. p. 1058-1063.

32. McCARTHY, Cavan Michael, PIMENTEL,Carlos Eduardo Pinto. Circulação com Micro-ISIS utilizando AUMIP: uma solução para bibliotecas de médio porte. . In: SEMINÁRIO SOBRE AUTOMAÇÃO DE BIBLIOTECAS E CENTROS DE DOCUMENTAÇÃO, 5, 1994, São José dos Campos. Anais... São José dos Campos : UNIVAP,1994. p. 87-94

33. McCARTHY, Cavan Michael. Pro-cite: um software para gerenciamento de bases de dados bibliográficos. Ci. Inf., Brasília, v.18,n.2, p.191-98, jul./dez. 1989.

34. McCARTHY, Cavan Michael. Uma base de dados para publicações locais. In: CONGRESSO BRASILEIRO DE BIBLIOTECONOMIA E DOCUMENTAÇÃO, 16, Salvador. Anais... Salvador: APBEB, 1991. p. 912-928.

35. MEDEIROS, Josete Soares. Leg- uma base de dados de legislação ambiental. Ci. Inf. Brasília, v.21, n.1, p.62-63, jan-/abr. 1992.

36. MEDINA, Evanise, ALBUQUERQUE, Maria Elizabeth B. C. de. SAB- Sistema de Automação de Biblioteca. In: ENCONTRO NACIONAL DE INFORMÁTICA E DOCUMENTAÇÃO JURIDICA, 2, Brasilia. Anais... Brasilia: ABDF/IBICT, 1986, p. 186-193.

37. MELO, Maria Aparecida de Oliveira, BRANDÃO, Maria Ricardina Moura, SANTOS, Paola De Marco Lopes de. Informatização da rede de Bibliotecas: a experiência da Secretaria Municipal de Cultura de São Paulo. In: SEMINÁRIO SOBRE AUTOMAÇÃO DE BIBLIOTECAS E CENTROS DE DOCUMENTAÇÃO, 5, 1994, São José dos Campos. Anais...São José dos Campos : UNIVAP, 1994. p. 65-72

38. MENDONÇA, Lúcia Maria Enout. MATOS, Márcia Maria. Produtos e serviços de informação no SEBRAE: as experiências no desenvolvimento de aplicativos em Micro-ISIS. Ci.Inf., v.20,n.1, p.8284,1991.

39. MIASHIRO, Wilson. As bases de dados operadas pela EMBRAPA. R. Bibliotecon. Brasília, v.13, n.2, p.:jun/dez 1985

40. MIKI, Hiroyuki. Miro-ISIS: uma ferramenta para o gerenciamento de bases de dados bibliográficas. Ciência da Informação, Brasília, v.18, n.1, p.3-14, jan./jun. 1989.

41. MORAES, Alice Ferry. Automação tatal da Biblioteca de Manguinhos com qualidade. In: SEMINÁRIO SOBRE AUTOMAÇÃO DE BIBLIOTECAS E CENTROS DE DOCUMENTAÇÃO, 6, 1997, Águas de Lindóia. Anais... São José dos Campos: INPE, 1997. p.134-139.

42. MOREIRA, José de Albuquerque. Informação musical: um projeto de banco de dados. In: CONGRESSO BRASILEIRO DE BIBLIOTECONOMIA E DOCUMENTAÇÃO, 16, Salvador, 1991. Anais... Salvador: APBEB, 1991. p.980-989.

43. NUNES, Claúdio O.I. et al. Automação dos serviços de informação na Universidade do Rio Grande e o desenvolvimento do sistema de administração de bibliotecas II - Sab-II. In: CONGRESSO BRASILEIRO DE BIBLIOTECONOMIA E DOCUMENTAÇÃO, 16, Salvador, 1991. Anais... Salvador: APBEB, 1991. p.990-1007.

44. OHIRA, Maria Lourdes Blatt, et al. Definição de uma base de dados para gravações de vídeo: utilização do MicrolSIS. In: PAINEL DE BIBLIOTECONOMIA EM SANTA CATARINA, 11, ENCONTRO DE USUÁRIOS DE MICROISIS DA REGIÃO SUL ,11, 1992, Florianópolis, Anais ... Florianópolis: UFSC/CED, 1993. p. 63-72.
45. OHIRA, Maria Lourdes Blatt. Automação de bibliotecas: utilização do MicrolSIS. In: PAINEL DE BIBLIOTECONOMIA EM SANTA CATARINA, 11, ENCONTRO DE USUÁRIOS DE MICROISIS DA REGIÃO SUL, 1,1992, Florianópolis, Anais ... Florianópolis: UFSC/CED, 1993. p. 23-31.

46. OHIRA, Maria Lourdes Blatt. Automação de bibliotecas: utilização do MicroISIS. Ci. Inf. v.21, n.3, p. 233-237, set./dez. 1992.

47. OHIRA, Maria de Lourdes Blatt. Biblionfo base de dados sobre automação em bibliotecas (informática documentária): 1986-1994. Ci. Inf. v. 23, n.3, p.369-371, set./dez. 1994.

48. OHIRA, Maria Lourdes B., MAIA, Maria Helena B. Maia, SELL, Maria Aparecida. Controle bibliográfico da produção intelectual dos profissionais da informação de Santa Catarina: Base de dados BIDAC (1976/1996). In: SEMINÁRIO SOBRE AUTOMAÇÃO DE BIBLIOTECAS E CENTROS DE DOCUMENTAÇÃO, 6, 1997, Águas de Lindóia. Anais... São José dos Campos: INPE, 1997. p.44-52.

49. OHIRA, Maria Lourdes B., SALVATO, Gilberto José. Utilização do CDS/ISIS no tratamento do acervo bibliográfico da produção técnico-científica da Fatma. SEMINÁRIO CATARINENSE DE BIBLIOTECONOMIA \& INFORMAÇÃO, 2, 1988. Florianópolis: Resumos... Florianópolis: SCB\&I, 1988.

50. OLIVEIRA, Stanley Robson de Medeiros, ANTUNES, João Francisco Gonçalves. AINFO: uma ferramenta para gerenciamento de bases de dados documentais e processos bibliográficos. In: SEMINÁRIO SOBRE AUTOMAÇÃO DE BIBLIOTECAS E CENTROS DE DOCUMENTAÇÃO, 6, 1997, Águas de Lindóia. Anais... São José dos Campos: INPE, 1997. p.181-186.

51. OlIVEIRA, Maria das Graças C. de, ARAÙJO, Rosa Maria Cardoso S. Sistema de informações seletivas especializadas (INFO-SEL\&S). R. Bibliotecon. Brasília, v.13, n. 1, p. 49-57, jan./jun. 1985.

52. OLIVEIRA NETO, Manuel Menezes, SCHRAMM, Mauro. ISIS-Magic: implementação de uma interface para tratamento de imagens no sistema MicrolSIS. In: CONGRESSO LATINO-AMERICANO DE BIBLIOTECONOMIA E DOCUMENTAÇÃO, 2, 1994, Belo Horizonte. Anais... Belo Horizonte: ABMG, 1994. p. 494-509.

53. PIMENTEL, Cléa Dubeux Pinto, PIMENTEL, Carlos Eduardo. Desenvolvimento de uma base de dados de usuários. In: SEMINÁRIO SOBRE AUTOMAÇÃO DE BIBLIOTECAS E CENTROS DE DOCUMENTAÇÃO, 5, 1994, São José dos Campos. Anais...São José dos Campos: UNIVAP, 1994. p.134-138.

54. RESMER, Maria José, COSTA, Olga Maria Soares. Conversão de bases de dados MicrolSIS para Internet. Ci.Inf., v.26, n.2, p.159-164, mai./ago. 1997

55. ROBREDO, Jaime. Dois novos sistemas com conmputadores para o processamento completo da informação documentária. R. Bibliotecon. Brasília, v.10, n. 1, p.69-72, jan./jun. 1892.

56. ROCHA, Sônia Correa da, MORETTI, Adri- 
ana Bueno. Base de dados de literatura periódica em ciências agrárias. In: SEMINÁRIO SOBRE AUTOMAÇÃO DE BIBLIOTECAS E CENTROS DE DOCUMENTAÇÃO, 6, 1997, Águas de Lindóia. Anais... São José dos Campos: INPE, 1997. p.187-189.

57. SÁ, Regina de Almeida, DONNARD, Heloisa Marie, DANTAS FILHO, Lourival Emílio. Uma base de dados bibliográficos em áreas sócio-econômicas. R. Bibliotecon. Brasília, v.13, n.2, p. 241-249, jul./dez. 1985.

58. SALVATO, Gilberto José. Informatização de documentos sob MicrolSIS: versatilidade com baixos custos. In: PAINEL DE BIBLIOTECONOMIA EM SANTA CATARINA, 11, ENCONTRO DE USUÁRIOS DE MICROISIS DA REGIÃO SUL, 1,1992, Florianópolis. Anais... Florianópolis: UFSC/ CED, 1993. p.55-56.

59. SANTOS , João Gomes dos. ABE, Naguiça. Utilização do micro CDS/ISIS: .a experiência do SENAI. Ci. Inf. v.20, n.1, jan/ jun., p. 74-77, 1991.

60. SANTOS, Gildenir Carolino, PASSOS, Rosemary. Desenvolvimento de base de dados em educação "EDUBASE", gereciado pelo software Micro-CDS/ISIS. In: SEMINÁRIO SOBRE AUTOMAĈ̃O DE BIBLIOTECAS E CENTROS DE DOCUMENTAÇÃO, 6, 1997, Águas de Lindóia. Anais... São José dos Campos: INPE, 1997. p.127-130.

61. SANTOS, Gildenir Carolino, GIANNONI, Maria Alice. Organização da hemeroteca sobre educação, gerenciada pelo software Micro CDS/ISIS na base de dados "HEMERED". In: SEMINÁRIO SOBRE AUTOMAÇÃO DE BIBLIOTECAS E CENTROS DE DOCUMENTAÇÃO, 6, 1997, Águas de Lindóia. Anais... São José dos Campos: INPE, 1997. p.131-133.

62. SANTOS, Gildenir Carolino dos, TRUZZI, Marilda, FERRONI, Egberto Luís. Gerenciador de biblioteca- GERBIBLI. In: SEMINÁRIO SOBRE AUTOMAÇÃO DE BIBLIOTECAS E CENTROS DE DOCUMENTA-

\section{Software for automation of libraries and documentation centers in Brazilian literature up to 1998}

\section{Abstract \\ This is an overall study about the utilization of software in automation of Brazilian libraries up to 1998. The paper analyzes the trends of this bibliographic production as to its authorship, type of documentation, means of publication, geographic distribution throughout the years. It also includes a succint description of the main software mencioned in the literature.}

\section{Keywords}

Software for libraries automation, Bibliographic databases managers bibliographic publication trends; Documentation.
ÇÃO, 5, 1994, São José dos Campos. Anais... São José dos Campos : UNIVAP,1994. p. 79-86.

63. SANTOS, Gildenir Carolino dos, PIETROSANTO, Ademir Giacomo. O acesso em base de dados em economia e educação, pela internet através da ferramenta WWWIsis. In: SEMINÁRIO NACIONAL DE BIBLIOTECAS UNIVERSITÁRIAS, 10, 1998, Fortaleza. Anais... (versão eletrônica: disquete).

64. SAYÃO, et al. Avaliação do processos de automação em bibliotecas universitárias. Transinformação, v. 1, n. 2, p. 233254, maio/ago. 1989.

65. SCHAUER, Maria Bernadete Câmara. Automação do centro de documentação da Price Waterhouse. In: CONGRESSO DE BIBLIOTECONOMIA, DOCUMENTAÇ̃̃O E CIÊNCIA DA INFORMAÇÃO, 1992, São Paulo. Anais... São Paulo: APB , 1992. p.267-275.

66. SELL, Maria Aparecida, SALVATO, Gilberto José. Base de dados bibliográficos "Libris" através do software MicrolSIS. In: CONGRESSO DE BIBLIOTECONOMIA, DOCUMENTAÇÃO E CIÊNCIA DA INFORMAÇÃO, 1992, São Paulo. Anais... São Paulo: APB, 1992. p. 276-83.

67. SIEBERT, Sérgio. SELL, Maria Aparecida. Base de dados de legislação estadual. In: PAINEL DE BIBLIOTECONOMIA EM SANTA CATARINA, 11, ENCONTRO DE USUÁRIOS DE MICROISIS DA REGIÃO SUL, 1, 1992, Florianópolis. Anais... Florianópolis: UFSC/CED, 1993. p.57-62

68. SILVA, Dirce Maris Nunes da. Circulação dirigida de periódicos - CDP, utilizando o software MicroISIS 2.3. In: : CONGRESSO DE BIBLIOTECONOMIA, DOCUMENTAÇÃO E CIÊNCIA DA INFORMAÇÃO, 1992, São Paulo. Anais... São Paulo: APB, 1992. p.189-98.

69. SILVA, Elenice Ávila da, CAREGNATO, Laís Freitas, et al. Automação do sistema de bibliotecas da Universidade Federal do Rio Grande do Sul. In: SEMINÁRIO SOBRE AUTOMAÇÃO DE BIBLIOTECAS E CENTROS DE DOCUMENTAÇÃO, 5, 1994, São José dos Campos. Anais...São José dos Campos: UNIVAP,1994 p. 110-113

70. SILVEIRA, Amélia. ARAÚJO, Franca Maria B. KNOLL, Marília D. Costa. Mini-Micro CDS/ISIS: uma proposta de aplicação no ensino da informação em Biblioteconomia e Ciência da Informação. R. Bras. Bibliotecon. e Doc., São Paulo: FEBAB, v.22., n.3/4, 1989.

71. SOLER, Renata Cristina de Almeida. Thesaurus e sistema automatizado de recuperação: a união que resulta qualidade. In: CONGRESSO DE BIBLIOTECONOMIA, DOCUMENTAÇÃO E CIÊNCIA DA INFORMAÇÃO, 1992, São Paulo. Anais... São Paulo: APB, 1992. p. 185-283.

72. SOUZA, Terezinha Batista de, CATARINO, Maria Elisabete, SANTOS, Paulo César dos. Metadados: catalogando dados na Internet. Transinformação, v.9, n.2, p.93105, maio/agosto, 1997.

73. SOUZA, Irene Josefa de, BELLÍZIA, Maria Helena Rosas. Catálogo de assunto au- tomatizado: a experiência da DIDC. In: SEMINÁRIO SOBRE AUTOMAÇÃO DE BIBLIOTECAS E CENTROS DE DOCUMENTAÇÃO, 3, 1989, Águas de Lindóia, São Paulo. Anais... Águas de Lindóia, 1989. p. 54-58.

74. TEIXEIRA, Ana Lúcia Drumond Alegria, GOMES, Ana Maria Campio. Os serviços bibliográficos automatizados da RFFSA. In: ENCONTRO NACIONAL DE INFORMÁTICA E DOCUMENTAÇÃO JURIDICA, 2 1986, Brasília. Anais... Brasília: IBDF/ IBICT, 1986. p.481-486.

75. ZANAGA, Mariângela Pisoni. Coversão Retrospectiva e cooperação no processamento técnico de materiais bibliográficos. In: SEMINÁRIO NACIONAL DE BIBLIOTECAS UNIVERSITÁRIAS, 8, 1994 , Campinas. Anais...Campinas: Unicamp, 1994. p.59-68

76. ZIVIANI, Nívio. Um sistema para recuperação eficiente de informação e textos. $R e$ vista Brasileira de Computação, Rio de Janeiro, v.5, n.3, p.3-15, jan./mar. 1990.

\section{Gercina Ângela Borém Lima}

Professora assistente da Escola de Biblioteconomia da UFMG. Mestre em Library and Information Science pela Clark Atlanta University, EUA. Coordenadora do projeto de pesquisa A Produção Bibliográfica Brasileira na Área de Tratamento e Tecnologia da Informação a partir de 1968.

E-mail: glima@eb.ufmg.br 